\title{
Efecto de la cobertura y de la fertilización en el desarrollo de plantas de Nothofagus alessandrii cultivadas en contenedor
}

\author{
Effect of cover and fertilization on the early development of Nothofagus alessandrii nursery \\ container seedlings
}

\author{
Rómulo Santelices $^{\text {a*, }}$, Rafael M Navarro Cerrillo ${ }^{b}$, Fernando Drake ${ }^{c}$, Carlos Mena ${ }^{\mathrm{d}}$ \\ *Autor de correspondencia: a Universidad Católica del Maule, Facultad de Ciencias Agrarias y Forestales, Departamento de Ciencias \\ Forestales, Centro de Desarrollo para el Secano Interior, casilla 617, Talca, Chile, tel.: 56-71-203500, fax: 56-71-203524, \\ rsanteli@ucm.cl \\ b Universidad de Córdoba, Facultad de Agronomía y Montes, Departamento de Ingeniería Forestal, Córdoba, España. \\ c Universidad de Concepción, Facultad de Ciencias Forestales, Departamento Manejo de Bosques y Medioambiente, \\ Concepción, Chile. \\ ${ }^{\text {d }}$ Universidad de Talca, Facultad de Ciencias Forestales, Centro de Geomática, Talca, Chile.
}

\author{
SUMMARY
}

Nothofagus alessandrii is an endangered species. Yet, available information for the propagation and nursery cultivation of this species is conspicuously lacking. To this end, we studied the effect of cover and fertilization on early growth of nursery seedlings over one growing season. Using a split plot experimental design with completely random fixed-effect blocks we monitored seedling growth with three levels of shade (plastic net Raschel ${ }^{\circledR}$ of 35, 50, y $80 \%$ ) and three doses of slow-release fertilizer (4, 6, and $10 \mathrm{~g}$ of Osmocote ${ }^{\circledR}$ per litter of growth media). Results demonstrated a significant effect of shade treatments on seedling development. Differences between seedlings treated with 35-50\% when compared with those treated with $80 \%$ cultivated were observed on the evaluated attributes. In general, fertilization did not affect seedling development. In conclusion, it is more efficient to cultivate N. alessandrii nursery seedlings at $35 \%$ shade and $4 \mathrm{~g} \mathrm{~L}^{-1}$ fertilization. Under this scenario, it is possible to produce plants, on average, with $28 \mathrm{~cm}$ in stem length; $4 \mathrm{~mm}$ of root collar diameter; $3.2 \mathrm{~g} \mathrm{plant}^{-1}$ total biomass; and quality indices of 6.9 (slenderness), 2.2 (rootshoot), and 2.1 (Dickson).

Key words: ruil, shadow, slow-release fertilizer.

\section{RESUMEN}

Nothofagus alessandrii es una especie en peligro de extinción, de la cual aún no hay información suficiente sobre el cultivo de plantas en vivero. Por ello, el objetivo de este estudio fue analizar el efecto de la cobertura y de la fertilización en el desarrollo inicial de las plantas durante una temporada. Utilizando un diseño en parcelas divididas en bloques al azar, se ensayaron tres niveles de sombra (malla Raschel ${ }^{\circledR}$ de 35, 50, y 80 \%) y tres dosis de fertilizante de entrega lenta (4, 6, y 10 g de Osmocote ${ }^{\circledR}$ por litro de sustrato). Los resultados muestran que hubo un efecto significativo de la sombra en el desarrollo de las plantas, observándose mejores atributos en plantas con 35-50 \% de sombra, respecto de las cultivadas con 80 \%. En general, la fertilización no afectó el desarrollo de las plantas. Se puede concluir que es más eficiente cultivar plantas de N. alessandrii en vivero bajo una cobertura de $35 \%$ de sombra y fertilizando con $4 \mathrm{~g} \mathrm{~L}^{-1}$. Así, es posible producir, en promedio, plantas de $28 \mathrm{~cm}$ de longitud de tallo, $4 \mathrm{~mm}$ de diámetro de cuello de la raíz, 3,2 g planta $^{-1}$ de biomasa total e índices de calidad de 6,9 para el de esbeltez, 2,2 para el de tallo-raíz y 2,1 para el de Dickson.

Palabras clave: ruil, sombra, fertilizante de entrega lenta.

\section{INTRODUCCIÓN}

Nothofagus alessandrii Espinosa (ruil), es una especie considerada en peligro crítico de extinción (UICN 2001). Una vía posible para cambiar su estado de conservación es la repoblación artificial, para lo cual es necesario conocer aspectos relacionados con el cultivo de plantas. Entre algunos de los factores de cultivo que deben considerarse, especialmente para regiones con climas mediterráneos, están el sombreo, la fertilización, y la micorrización (Villar-Salvador 2003, Rodríguez 2008).

En algunas especies es importante el empleo de cubiertas que protejan a las plantas, por ejemplo, de una radiación demasiado elevada (Landis et al. 1995). Para algunos Nothofagus spp. existe evidencia de la importancia de utilizar sombra en el desarrollo del cultivo (Santelices 
et al. 1995, Donoso et al. 2006ab). En particular, se ha observado (datos sin publicar) una alta mortalidad en vivero al cultivar plantas de $N$. alessandrii a plena luminosidad. Existen diferentes medios para proteger las plantas del exceso de radiación, siendo en la actualidad las mallas de sombra ampliamente aceptadas (Landis et al. 1995).

Una práctica cultural importante en vivero es la fertilización, debido a que incide directamente en el crecimiento de las plantas, mejora su posterior capacidad de arraigamiento en el transplante, e incrementa la resistencia al estrés biótico y abiótico (Oliet et al. 2005). Uno de los métodos empleados actualmente es el que utiliza fertilizantes de entrega lenta, que se caracterizan por suministrar los elementos minerales de forma gradual en el tiempo. Con él se logra mejorar la eficiencia en el aprovechamiento de los nutrientes y disminuir las pérdidas por lixiviación (Fernández-Escobar et al. 2004).

La información sobre el cultivo de plantas de $N$. alessandrii es reducida y muestra resultados variables (San Martín et al. 2006, Santelices et al. 2009a). Se sabe que al aumentar la cobertura, también lo hace la supervivencia de las plantas en vivero (Olivares et al. 2005). Respecto de la fertilización, el empleo de diferentes dosis de Osmocote ${ }^{\circledR}$ no tiene un claro efecto en el desarrollo de las plantas, ya sea evaluando algunos atributos morfológicos o índices de calidad (Santelices et al. 2009ab). En consecuencia, resulta de interés estudiar y comparar el efecto de diferentes concentraciones de este fertilizante en la producción de plantas en vivero.

No se encontraron estudios que analicen en forma conjunta el efecto de la sombra como medio para modificar las condiciones ambientales y la fertilización en el cultivo de plantas de $N$. alessandrii. Tanto la sombra como la fertilización debieran afectar significativamente el desarrollo inicial de las plantas de $N$. alessandrii. Se espera que al aumentar el nivel de sombra disminuya el crecimiento y que con mayores dosis de fertilización éste aumente. El objetivo de esta investigación es analizar el efecto de la sombra y distintas dosis de fertilización en el crecimiento y respuesta funcional de brinzales cultivados en contenedor.

\section{MÉTODOS}

Material vegetal y cultivo. Las semillas utilizadas fueron de la procedencia Lo Ramírez (35 $10^{\circ}$ S y $72^{\circ} 06^{\prime} \mathrm{O}$, $385 \mathrm{~m}$ de elevación). En promedio, tuvieron una pureza del 80,3 $\pm 1,7 \%$, el peso de 1.000 semillas fue de 10,59 \pm 0,25 g; y la viabilidad fue de un 6,1 $\pm 0,13 \%$.

El ensayo se llevó a cabo en el vivero de la Universidad Católica del Maule ( $35^{\circ} 26^{\prime}$ 09” S y 71³7’ 13” O, 131 m de elevación). La latencia de las semillas fue superada sumergiéndolas durante 24 horas en una solución de $\mathrm{GA}_{3}\left(\right.$ Giberplus $^{\circledR}$ ) a $200 \mathrm{mg} \mathrm{L}^{-1}$ y la siembra se efectuó el 21 de septiembre de 2007. Una vez iniciada la germinación (después de 10 días), las semillas se sembraron en contenedores de plástico rígido de 140 mL (Termomatrices $\left.^{\circledR}\right)$. Como sustrato se utilizó una mezcla de corteza compostada de pino y perlita $8(7: 3 \mathrm{v} / \mathrm{v})$, que fue mezclada con el fertilizante Osmocote ${ }^{\circledR} 18-6-12$, en dosis de 4, 6, y $10 \mathrm{~g} \mathrm{~L}^{-1}$. Durante el cultivo, las plantas fueron protegidas con una malla plástica de sombra $\left(\right.$ Raschel $\left.^{\circledR}\right)$ de un 35, 50, y 80 \% de reducción de luz, de acuerdo a los niveles determinados para el ensayo. El riego se realizó utilizando microaspersores y un programador, manteniendo el sustrato a capacidad de campo.

Diseño experimental y mediciones. El ensayo fue planteado con un diseño estadístico en parcelas divididas en bloques completamente aleatorizados de efectos fijos. Se realizaron tres réplicas por tratamiento, con 20 plantas por unidad experimental. La parcela completa fue representada por el factor sombra, mientras que la subparcela por el de fertilización.

Transcurrida una temporada en vivero (a comienzos de mayo de 2008), se midieron los atributos morfológicos longitud del tallo (L) y diámetro de cuello (DCR) de todas las plantas. Además, se seleccionaron aleatoriamente nueve plantas por tratamiento y se les midió la biomasa aérea (BA), biomasa de la raíz (BR) y biomasa total (BT). Con esta información se calcularon los índices de esbeltez (IE), de tallo/raíz (ITR), y de Dickson (ID), de acuerdo a las fórmulas [1], [2] y [3], respectivamente.

$$
\begin{aligned}
& I E=\frac{L(\mathrm{~cm})}{D C R(\mathrm{~mm})} \\
& \text { ITR }=\frac{B A(\mathrm{~g})}{B R(\mathrm{~g})} \\
& \text { ID }=\frac{\mathrm{B} \mathrm{T}(\mathrm{g})}{\frac{\mathrm{cm})}{D C R(\mathrm{~mm})}+\frac{B A(\mathrm{~g})}{B R(\mathrm{~g})}}
\end{aligned}
$$

Análisis de los datos. Los análisis de varianza y las comparaciones de medias, se realizaron usando el procedimiento GLM del programa estadístico SPSS para Windows V. 15. Los valores medios que presentaron diferencias significativas fueron comparados con la prueba de Tukey $(P \leq 0,05)$.

\section{RESULTADOS}

No se observó una interacción entre los factores estudiados, por lo cual los resultados de los factores principales se muestran por separado.

Se registraron diferencias significativas en todos los atributos de las plantas de $N$. alessandrii sometidas a diferentes grados de cobertura, a excepción del índice tallo/raíz (cuadro 1). En general, a medida que aumentó la sombra, el crecimiento fue menor tanto en diámetro como 
en altura, lo que también se vio reflejado en los índices de calidad, observándose diferencias significativas entre aquellas plantas tratadas con 35-50 \% de sombra respecto de aquellas cultivadas con $80 \%$. La fertilización no afectó a ninguno de los atributos evaluados, observándose un desarrollo homogéneo de las plantas (cuadro 1).

\section{DISCUSIÓN}

Las plantas que disponen de mayor radiación presentan un crecimiento mayor, lo que se evidencia en la producción de biomasa de las plantas crecidas con una sombra del 35 y 50 \% (cuadro 1). Ello, probablemente, porque con tales niveles de sombra la fotosíntesis fue mayor (Taiz y Zeiger 2006). Cuando factores como la luz y la humedad del suelo están en condiciones favorables, las plantas alcanzan las máximas tasas fotosintéticas. Si uno de esos factores llega a ser limitante (como el exceso de sombra), las plantas reducen su tasa fotosintética (Martínez et al. 2007).

Los índices tallo/raíz y de esbeltez de las plantas cultivadas con sombra moderada (35-50 \%) muestran un equilibrio entre el crecimiento de la parte aérea y el de la radical. Es decir, entre la superficie que transpira y la que absorbe agua y, de acuerdo a Villar-Salvador (2003), podrían mantener un mejor estado hídrico con un consumo más moderado de agua en situaciones de deficiencia hídrica. Si bien es cierto que con todos los niveles de cobertura ensayados se alcanzaron las exigencias mínimas sugeridas por INN (2005) para Nothofagus nervosa (Phil.) Dim. et Mil. (longitud del tallo de 25 - $40 \mathrm{~cm}$, diámetro de cuello de la raíz $>3 \mathrm{~mm}$ e índice de esbeltez $<8,3$ ), las plantas con sombra de 35 - $50 \%$ tienden a ser más equilibradas que las cultivadas con un $80 \%$ de cobertura.

El nulo efecto de las diferentes dosis de fertilizante concuerda con lo señalado por Santelices et al. (2009b) para esta misma especie. No obstante, los atributos de las plantas son diferentes entre los dos ensayos, lo que podría estar explicado por dos factores: la época de siembra y las condiciones ambientales entre ambos ensayos fueron diferentes. Al igual que con los tratamientos de sombra, el tamaño de las plantas cumple con el mínimo exigido por la normativa establecida para $N$. nervosa (INN 2005). $\mathrm{Al}$ aumentar la concentración del fertilizante, no se registraron variaciones significativas en el crecimiento, lo que indicaría que no se alcanzó el nivel de toxicidad descrito por Salifu y Jacobs (2006).

Al contrastar los resultados con los presentados por Bustos et al. (2008), queda de manifiesto que N. alessandrii tiene un respuesta diferente al de otras especies de su género y podría alcanzar un nivel óptimo de crecimiento con menos requerimientos nutricionales, gracias a su eficiencia en el uso de los nutrientes (San Martín et al. 2006). En este contexto, es posible que no se hayan encontrado diferencias significativas en los distintos atributos evaluados debido a que, de acuerdo a la ley del mínimo de Liebeg, las plantas no tuvieron un factor limitante nutricional para su crecimiento. Por otra parte, la planta podría responder ante el mayor suministro de nutrientes almacenando lo que podría considerarse un exceso en diferentes órganos, tal como sucede, por ejemplo, con Quercus petraea Liebl. (Berger y Glatzel 2001) o Quercus ilex L. (El Omari et al. 2003), y removilizarlos posteriormente tal como lo señalan estos últimos autores.

\section{CONCLUSIONES}

La sombra afecta al desarrollo de las plantas de $N$. alessandrii cultivadas en vivero durante una temporada.

Cuadro 1. Efecto de la sombra y la fertilización en los atributos morfológicos e índices de calidad de plantas de Nothofagus alessandrii cultivadas en vivero durante una temporada (media \pm error estándar; $\mathrm{n}=20$ ).

Effect of shade and fertilization on morphological attributes and quality indices of Nothofagus alessandrii nursery seedlings cultivated during one growing season (media \pm standard error; $n=20$ ).

\begin{tabular}{|c|c|c|c|c|c|c|c|c|}
\hline \multirow[t]{2}{*}{ Tratamiento } & \multirow{2}{*}{$\begin{array}{c}\mathrm{L} \\
(\mathrm{cm})\end{array}$} & \multirow{2}{*}{$\begin{array}{l}\text { DCR } \\
(\mathrm{mm})\end{array}$} & \multicolumn{3}{|c|}{ Biomasa (g) } & \multicolumn{3}{|c|}{ Índice de calidad } \\
\hline & & & Aérea & Radical & Total & Esbeltez & Tallo/Raíz & Dickson \\
\hline \multicolumn{9}{|l|}{ Sombra: } \\
\hline $35 \%$ & $27,9 \pm 0,8 a$ & $4,0 \pm 0,06 a$ & $2,2 \pm 0,17 a$ & $1,00 \pm 0,07 a$ & $3,19 \pm 0,19 a$ & $6,9 \pm 0,15 a$ & $2,2 \pm 0,4 a$ & $2,1 \pm 0,30 a$ \\
\hline $50 \%$ & $31,8 \pm 0,7 a$ & $4,0 \pm 0,06 a$ & $2,3 \pm 0,19 a$ & $1,07 \pm 0,07 a$ & $3,39 \pm 0,25 a$ & $7,9 \pm 0,14 b$ & $2,2 \pm 0,3 a$ & $1,9 \pm 0,25 a$ \\
\hline $80 \%$ & $25,2 \pm 0,7 b$ & $3,2 \pm 0,08 b$ & $1,6 \pm 0,15 b$ & $0,56 \pm 0,04 b$ & $2,19 \pm 0,19 b$ & $8,0 \pm 0,30 \mathrm{~b}$ & $3,0 \pm 0,3 a$ & $1,0 \pm 0,22 b$ \\
\hline \multicolumn{9}{|c|}{ Dosis fertilizante: } \\
\hline $4 \mathrm{~g} \mathrm{~L}^{-1}$ & $28,4 \pm 0,7 a$ & $3,8 \pm 0,07 a$ & $1,93 \pm 0,23 a$ & $0,87 \pm 0,12 \mathrm{a}$ & $2,81 \pm 0,32 a$ & $7,4 \pm 0,13 \mathrm{a}$ & $2,3 \pm 0,5 a$ & $1,6 \pm 0,55 a$ \\
\hline $6 \mathrm{~g} \mathrm{~L}^{-1}$ & $29,7 \pm 0,8 a$ & $3,9 \pm 0,07 a$ & $2,00 \pm 0,17 a$ & $0,83 \pm 0,10 a$ & $2,83 \pm 0,24 a$ & $7,7 \pm 0,15 a$ & $2,6 \pm 0,4 a$ & $1,5 \pm 0,44 a$ \\
\hline $10 \mathrm{~g} \mathrm{~L}^{-1}$ & $28,7 \pm 0,9 a$ & $3,8 \pm 0,09 a$ & $2,20 \pm 0,14 a$ & $0,92 \pm 0,08 a$ & $3,12 \pm 0,20 a$ & $7,7 \pm 0,30 \mathrm{a}$ & $2,5 \pm 0,3 a$ & $1,8 \pm 0,23 a$ \\
\hline
\end{tabular}

$\mathrm{L}=$ longitud del tallo, $\mathrm{DCR}=$ diámetro de cuello de la raíz. Valores promedios con la misma letra no difieren significativamente entre sí, $P>0,05$. $\mathrm{L}=$ stem length, $\mathrm{DCR}=$ root collar diameter. Mean values with the same letter are not significantly different, $P>0.05$. 
Con niveles de sombra moderada (35 - 50 \%) se obtienen plantas de calidad funcional aceptable para la forestación, de acuerdo a sus atributos morfológicos e índices de calidad. No se observa un efecto en el desarrollo de las plantas como consecuencia de la aplicación de diferentes dosis del fertilizante de entrega lenta. Los atributos morfológicos y los índices de calidad indican que son plantas aptas para la forestación. Por ello, sería más eficiente cultivar plantas de $N$. alessandrii en vivero bajo una cobertura de $35 \%$ de sombra y fertilizando con $4 \mathrm{~g} \mathrm{~L}^{-1}$ de fertilizante de entrega lenta.

\section{REFERENCIAS}

Berger TW, G Glatzel. 2001. Response of Quercus petraea seedlings to nitrogen fertilization. Forest Ecology and Management 149: 1-14.

Bustos F, M González, P Donoso, V Gerding, C Donoso, B Escobar. 2008. Efectos de distintas dosis de fertilizante de liberación controlada (Osmocote ${ }^{\circledR}$ ) en el desarrollo de plantas de coigüe, raulí y ulmo. Bosque 29(2): 155-161.

Donoso P, C Donoso, P Marchelli, L Gallo, B Escobar. 2006a. Nothofagus nervosa (Phil.) Dim. et Mil. Otros nombres científicos usados: Nothofagus alpina, Nothofagus procera. Raulí. In Donoso C ed. Las especies arbóreas de los bosques templados de Chile y Argentina, autoecología. Valdivia, Chile. Marisa Cuneo Ediciones. p 448-461.

Donoso P, C Donoso, L Gallo, M Azpilicueta, A Baldini, B Escobar. 2006b. Nothofagus obliqua (Mirb.) Oerst. Roble, Pellín, Hualle. In Donoso C ed. Las especies arbóreas de los bosques templados de Chile y Argentina, autoecología. Valdivia, Chile. Marisa Cuneo Ediciones. p: 471-485.

El Omari B, X Aranda, D Verdaguer, G Pascual, I Fleck. 2003. Resource remobilization in Quercus ilex L. resprouts. Plant and Soil 252: 349-357.

Fernández-Escobar R, Benlloch M, E. Herrera E, JM García-Novelo. 2004. Effect of traditional and slow-release $\mathrm{N}$ fertilizers on growth of olive nursery plants and $\mathrm{N}$ losses by leaching. Scientia Horticulturae 101(1-2): 39-49.

INN (Instituto Nacional de Normalización, CL). 2005. NCH2957/0 Madera. Material de propagación de uso forestal. Parte 0: producción y comercialización. Santiago, Chile. INN (Instituto Nacional de Normalización). 13 p.

Jiménez MD, M Pardos, J Puértolas, LA Kleczkowski, JA Pardos. 2004. Deep shade alters the acclimation response to moderate water stress in Quercus suber L. Forestry 82(3): 285-298.

Landis TD, RW Tinus, SE McDonald, JP Barnett. 1995. Nursery Planning, Development, and Management. Vol. 1. The Container Tree Nursery Manual. Agric. Handbook 674. Washington, DC. U.S. Department of Agriculture, Forest Service. $188 \mathrm{p}$.

Martínez G, MV Lencinas, PL Peri, M Arena. 2007. Photosyn- thetic plasticity of Nothofagus pumilio seedlings to light intensity and soil moisture. Forest Ecology and Management 243(2-3): 274-282.

Navarro-Cerrillo RM, P Villar-Salvador, A Del Campo. 2006. Morfología y establecimiento de los plantones. In Cortina J, JL Peñuelas, J Puértolas, R Savé, A Vilagrosa eds. Calidad de planta forestal para la restauración en ambientes mediterráneos, estado actual de conocimientos. España. Ministerio del Medioambiente, Dirección general para la biodiversidad. p. 67-88.

Oliet J, R Planelles, F Artero, DF Jacobs. 2005. Nursery fertilization and tree shelters affect long-term field response of Acacia salicina Lindl. planted in Mediterranean semiarid conditions. Forest Ecology and Management 215(1-3): 339-351.

Olivares P, J San Martín, R Santelices. 2005. Ruil (Nothofagus alessandrii): Estado del conocimiento y desafíos para su conservación. Talca, Chile. Departamento de Protección de Recursos Naturales. Editorial de la Comisión Nacional del Medioambiente (CONAMA). 55 p.

Rodríguez D. 2008. Indicadores de calidad de planta forestal. México. Mundi Prensa. 156 p.

Salifu KF, DF Jacobs. 2006. Characterizing fertility targets and multi-element interactions in nursery culture of Quercus rubra seedlings. Annals of Forest Science 63(1): 231-237.

San Martín J, R Santelices, R Henríquez. 2006. Nothofagus alessandrii Espinosa, Ruil. Familia: Fagaceae. In C. Donoso ed. Las especies arbóreas de los bosques templados de Chile y Argentina, autoecología. Valdivia, Chile. Marisa Cuneo Ediciones. p. 390-400.

Santelices R, L Herrera, J Osores. 1995. Cultivo en vivero del hualo (Nothofagus glauca (Phil.) Krasser) bajo diferentes gradientes de luminosidad y espaciamiento. Ciencias Forestales (Chile) 10(1-2): 3-13.

Santelices R, RM Navarro, F Drake. 2009a. Caracterización del material forestal de reproducción de cinco procedencias de Nothofagus alessandrii Espinosa, una especie en peligro de extinción. Interciencia 34(2): 113-119.

Santelices R, RM Navarro, F Drake. 2009b. Propagation and seedling cultivation of the endemic species Nothofagus alessandrii Espinosa in Central Chile. Restoration Ecology DOI: 10.1111/j.1526-100X.2009.00550.x.

Taiz L, E Zeiger. 2006. Plant physiology. $4^{\text {th }}$ edition. Sunderland, MA, USA. Sinauer Associates. 764 p.

UICN (Unión Internacional para la Conservación de la Naturaleza, SZ, UK). 2001. Categorías y criterios de la lista roja de la UICN: Versión 3.1. Comisión de Supervivencia de Especies de la UICN. Gland, Suiza y Cambridge, Reino Unido. $33 \mathrm{p}$.

Villar-Salvador P. 2003. Importancia de la calidad de planta en los proyectos de revegetación. In Rey-Benayas JM, T Espigares-Pinilla, JM Nicolau-Ibarra eds. Restauración de ecosistemas mediterráneos. Alcalá de Henares, España. Universidad de Alcalá. p. 65-86. 